\title{
Licochalcone A Induces Cholangiocarcinoma Cell Death Via Suppression of Nrf2 and NF- $\kappa$ B Signaling Pathways
}

\author{
Phatthamon Laphanuwat*, Sarinya Kongpetch, Laddawan Senggunprai, \\ Auemduan Prawan, Veerapol Kukongviriyapan
}

\begin{abstract}
Objective: To investigate the anti-tumor effect of licochalcone A (LCA) on proliferation and migration in cholangiocarcinoma (CCA) cells and to elucidate their underlying mechanisms. Methods: Human CCA cells, KKU-100, KKU-213, KKU-214, KKU-156, and KKU-452 were used to study effect of LCA on proliferation and migration by a cytotoxicity assay, wound healing assay. Reactive oxygen species levels were evaluated using DHE-fluorescent probes. Proteins associated with cancer survival and progression were analyzed by immune blotting assay. Results: LCA suppressed proliferation and induced cell death in CCA cells including KKU-100, KKU-213, KKU-214, KKU-156, and KKU-452. The CCAs cells were suppressed in association with LCA-induced accumulation of intracellular reactive oxygen species (ROS). Increased formation of ROS was causally related with suppression of Nrf2 and its down-stream antioxidant and cytoprotective enzymes. These effects may lead to the expression of Bax and release of cytochrome $\mathrm{c}$ and ensuring cell death. Interestingly, LCA could also inhibit cell migration and cell cycle arrest at low concentrations. These effects were associated with down-regulation of NF-kB, STAT3 and their down-stream proteins, cyclin D1, VEGF, and ICAM-1. Conclusions: These results suggest that LCA has potential therapeutic activity in suppression of CCA cells.
\end{abstract}

Keywords: Cholangiocarcinoma- licochalcone A- anti-cancer- reactive oxygen species- anti-migration

Asian Pac J Cancer Prev, 23 (1), 115-123

\section{Introduction}

Biliary duct cancer or cholangiocarcinoma (CCA) is a rare and lethal cancer where the incidence is increasing worldwide. CCA is found frequently in some regions in the world, including Southeast Asia countries, and South China (Zabron et al., 2013). The etiology of CCA, however, is still not firmly determined. Clearly, incidence of CCA varies widely according to geographical regions, suggesting the impact of different sets of major risk factors including chronic infection of Opisthorchis spp and Clonorchis spp, primary sclerosing cholangitis, hepatolithiasis, hepatitis and life styles (Tyson and El-Serag, 2011). Liver fluke infection is a major risk factor of CCA in the endemic area of liver fluke in Southeast Asian countries, South China and Korea (Khan et al., 2019). In non-endemic area of liver fluke infection, other factors may contribute to the risk of CCA. The potential causes of non-liver fluke associated cholangiocarcinoma development are related with life styles or occupational contact to certain chemical agents, long term infection from hepatitis viral (Lee et al., 2009; Kubo et al., 2017). A gradual and sustaining exposure to these carcinogens slowly transform epithelial cells into malignancies without showing obvious symptoms to most of the patients until their 50s to 70s. Therefore, patients are generally diagnosed at their late stage of the disease (Doherty et al., 2017). The molecular carcinogenesis of CCA is orchestrated by a complex interplay of several oncogenic pathways. Previous studies classified CCA into two subtypes including inflammatory- and proliferationdriven subtypes. Each one has prominent pathways that are prerequisite for cancer initiation and survival (Banales et al., 2020). Treatment options for cholangiocarcinoma including surgical resection, radiation therapy, and chemotherapy. Among these, complete surgical resection is the only option that offers a curative potential. Unfortunately, most of CCA patients are diagnosed at the late stage of the disease when chemotherapy mostly becomes the only option for the patients. Gemcitabine and platinum-based therapy has been used as a first-line drugs to treat biliary tract carcinoma (Doherty et al., 2017). However, drug treatment does provide only a modest survival benefit, because of the development of resistance (Banales et al., 2020).

The alternative treatments for cholangiocarcinoma which comprised of targeted therapy, immunologicalbased therapy, and peptide-based vaccine have been 
attempted (Al-Rajabi and Sun, 2021). Medicinal plants are rich sources of therapeutic agents. A variety of phytochemical substances have led to some interesting lead compounds as possible therapeutic drugs. Many classes of phytochemicals show a variety of pharmacological activities including anticancer.

Here, a new chalcone-based flavonoid compound extracted from licorice root, namely Licochalcone A (LCA) was investigated. Licorice is one of the most commonly used in herbal medicine preparations based on its neutralization effects that could give a synergistic effect to other constituents (Liu et al., 2019). Previous reports showed protective effects of LCA on a number of inflammatory models in animals including galactosamineinduced liver injury and ulcerative colitis through up-regulation of $\mathrm{Nrf} 2$ and down-regulation of NF- $\mathrm{KB}$ (Lv et al., 2019; Liu et al., 2021). Nevertheless, LCA exerted an antiproliferative effect on lung cancer cells by suppression of Nrf2 expression via miR-144 (Chen et al., 2018) and oral cancer cells by a decreased expression of NF-KB (Shen et al., 2014). As Nrf2 is a master of cellular antioxidant defense, the effect of LCA on the role of Nrf2 in CCA cells is crucial and still unknown.

Moreover, LCA shows a number of pharmacological activities such as anti-inflammation, anti-bacterial, and anti-aging (Fu et al., 2013; Gatta Daniela Maria et al., 2019; Ze-Feng et al., 2020). There were only few studies showing the anticancer activity and its mechanism of action. The present study was to investigate the antitumor activity and the underlying mechanism of LCA in CCA cells. It was found that LCA suppressed cholangiocarcinoma cell proliferation and induced cell death, where the effects were associated with induction of intracellular reactive oxygen species (ROS) accumulation and suppression of Nrf2 and NF- $\mathrm{KB}$ expression. Moreover, LCA could also inhibited cell migration. These results suggest the potential therapeutic activity of LCA in cholangiocarcinomas.

\section{Materials and Methods}

\section{Chemicals and reagents}

Licochalcone A was purchased from Medchem Express (Middlesex, NJ, USA). Ham's F12 nutrient mixture media was product of Gibco BRL Life Technologies (Grand Island, NY, USA). Fetal bovine serum was from Himedia (Mumbai, India, Cat. No. RM9955). Dihydroethidium (DHE)-fluorescence probes, sulforhodamine B (SRB), propidium iodide (PI) were from Sigma Aldrich (St. Louis, MO, USA). Antibodies against cytochrome c, Bax, Bcl-xL, Nrf2, GCLC, NF- $\kappa$ B, cyclin D1, VEGF, ICAM-1, $\beta$-actin and the secondary antibodies of goat anti-mouse IgG-horseradish peroxidase (HRP), goat anti-rabbit IgG-HRP, and mouse anti-goat IgG-HRP were purchased from Santa Cruz Biotechnology (Dallas, TX, USA). The antibody against HO-1 was obtained from Enzo Life Sciences (Farmingdale, NY, USA). The Antibody against STAT3 was from Cell signaling (Danvers, MA, USA). The antibody against GAPDH was from Affinity Biosciences (Cincinnati, OH, USA).

\section{Cell lines and cell culture}

The human cholangiocarcinoma (CCA) cell lines, KKU-100, KKU-213, KKU-214, KKU-M156 and KKU-452 were kindly provided by the Cholangiocarcinoma Research Institute, Khon Kaen university, Thailand. Cell line authentication was done by short tandem repeat (STR) profiling at the cell line bank indicated. The mycoplasma-free cells were cultured in Ham's F12 media supplemented with $10 \mathrm{mM} \mathrm{N}$-2-hydroxyethylpiperazine$\mathrm{N}^{\prime}$-2-ethanesulfonic acid (HEPES), pH 7.3, gentamicin sulfate $(50 \mu \mathrm{g} / \mathrm{ml})$, penicillin (100 unit $/ \mathrm{ml})$, and $10 \%(\mathrm{v} / \mathrm{v})$ fetal bovine serum (FBS).

\section{Cytotoxic testing}

Cells were seeded at the density of 5,000 cells/well onto 96-well plates and incubated overnight before treatment with various concentrations of LCA for $72 \mathrm{~h}$. Cultured cells were fixed with $10 \%$ trichloroacetic acid (TCA), stained with $0.4 \%$ SRB, then excess dye was washed with $1 \%$ acetic acid. Protein bound dye was solubilized with 10 $\mathrm{mM}$ TRIS buffer, $\mathrm{pH} 10.5$ for absorbance reading with a microplate reader at a wavelength of $540 \mathrm{~nm}$.

\section{Cell death assay}

Cytotoxic and apoptotic cells were detected by acridine orange and ethidium bromide (AO/EB). Briefly, cells were seeded in 96-well plates overnight. The cells were treated with LCA at indicated concentrations for $72 \mathrm{~h}$. The media was removed, and the cells were stained with $0.1 \mathrm{mg} /$ $\mathrm{ml}$ AO/EB dye. The cells were examined under a Nikon eclipse TS100 inverted fluorescent microscope with B2A filter. Living cells have a uniformly green nucleus with an organized cell structure. Early apoptotic cells have a bright green or orange nucleus with condensed or fragmented chromatin. Late apoptotic cells lose membrane integrity and show condensed and fragmented orange chromatin. Images of the cells were captured at predetermined areas, and the number of cells in each stage were counted using CellProfiler software (v 4.1.3, Broad Institute, USA).

\section{Wound healing assay}

KKU-100 cells at density of 100,000 cells/well were seeded into a 24-well plate overnight. A scratched wound was created by a small pipette tip. The cells were treated with different concentrations of LCA, and images of wound gap were captured at after 0-72 h. Cell migration, as measured by closure of the wound gap, was analyzed using CellProfiler software.

\section{Cell cycle analysis}

Cells were seeded at a density of 500,000 cells/well onto a 6-well plate overnight. After treated with LCA, cells were then fixed with $70 \%$ cold ethanol for overnight and permeabilized with $0.1 \%$ Triton X-100. DNA contents were stained with PI solution for 15 min before detection by flow cytometer (FACSCalibur, Becton, Dickinson and Company). Samples were collected 20,000 events and analyzed using FlowJo 10.7.1 (Tree Star Inc., Ashland, OR, USA). 
Reactive oxygen species (ROS) detection

ROS measurement was performed in 96-well plates. Cells were seeded at a density of 8,000 cells/well. After incubated with LCA for $24 \mathrm{~h}$, cells were washed with PBS, and DHE-fluorescence probes in serum-free media were added at a final concentration of $5 \mu \mathrm{M}$. Plates were incubated at $37^{\circ} \mathrm{C}$ for $30 \mathrm{~min}$, cells were then washed with PBS and immediately analyzed with a fluorescent microscope (Ex/Em: 535/590 nm).

\section{Western blot analysis}

Thirty micrograms of protein from whole cell lysates were separated on SDS-PAGE, then transferred to a nitrocellulose membrane, and blocked with 3\% BSA. The blotting membrane was incubated overnight at $4{ }^{\circ} \mathrm{C}$ with indicated antibodies. Detection of the specific proteins was done by the chemiluminescent method using the ChemiDoc MP imaging system (BioRad).

\section{Statistical analysis}

A comparison between two groups was performed using two-way ANOVA. $\mathrm{IC}_{50}$ values were derived by a sigmoidal dose-response (variable slope) curve using GraphPad Prism 7 software. Experiments were performed in biological triplicates. Positive DHE cells were counted in a total number of at least 1,000 cells from 4 different fields. Each bar represents the mean \pm s.d. The level of statistical significance was determined as $* p \leq 0.05$, $* * \mathrm{p} \leq 0.01$ and $* * * \mathrm{p} \leq 0.001$.

\section{Results}

Licochalcone A suppresses cholangiocarcinoma cells viability and proliferation

Licochalcone A (LCA) is a chalcone compound extracted from roots of Glycyrrhiza spp. or licorice (Figure 1a). The effects of LCA on viability and proliferation of CCA cells were investigated. The cytotoxic effects of LCA in several lines of CCAs from different histological types (KKU-100, KKU-156, KKU-213, KKU-214, and KKU-452) were firstly screened. CCA cell proliferation and viability are dose dependently inhibited following 72 $\mathrm{h}$ treatment with LCA (Figure 1b). The cytotoxic effects of LCA are shown as the median inhibitory concentration (IC50) (Figure 1c). Each individual cell line is slightly different in susceptibility to LCA judged by the $\mathrm{IC}_{50}$ values. The responses of the cells could be grouped into 3 groups; the high responsive cells (KKU-213), the intermediate responsive cells (KKU-156, KKU-214, KKU452), and the low responsive cells (KKU-100). In the following experiments the high and low responsive cells were used.

Licochalcone A induces G2/M arrest and apoptosis cell death in cholangiocarcinoma cells

To further investigate whether LCA induced cholangiocarcinoma cell cycle arrest and cell death, the cells were firstly treated with various concentrations of LCA for 24 and $48 \mathrm{~h}$ and proceeded to the cell cycle analysis using propidium iodide (PI) staining. The results showed that, at the early time point of $24 \mathrm{~h}$, all CCA cells were arrested at a high concentration of LCA at G2/M phase with a slightly higher $\mathrm{G} 2 / \mathrm{M}$ proportion in highly responsive KKU-213 cells. The G2/M phase arrests were more pronounced after $48 \mathrm{~h}$ of treatment. (Figure $2 \mathrm{a}, \mathrm{b}$ ).

Not only did cell cycle distribution change upon LCA treatment, but the morphology of the cells also changed by the cells acquiring a smaller size and more granularity with irregular shapes when observed under the brightfield microscope (Figure 2c).

In an analysis of LCA induced apoptotic cell death, a large proportion of the cells were subjected to apoptosis after $72 \mathrm{~h}$ of the treatments (Figure 3a, b) analyzed by the acridine orange/ethidium bromide staining. The number of early and late apoptotic cells were varied among CCA cells (Figure 3b) indicating the different rates in cell death induction in each cell type.

To examine the cell death pathway, proteins involving with apoptosis were analyzed by Western immunoblotting. LCA induced an increase of pro-apoptotic protein Bax and a decline of an anti-apoptotic protein Bcl-xL. Moreover, accumulation of mitochondrial cytochrome $\mathrm{c}$ in both KKU-213 and KKU-100 cells suggested the implication

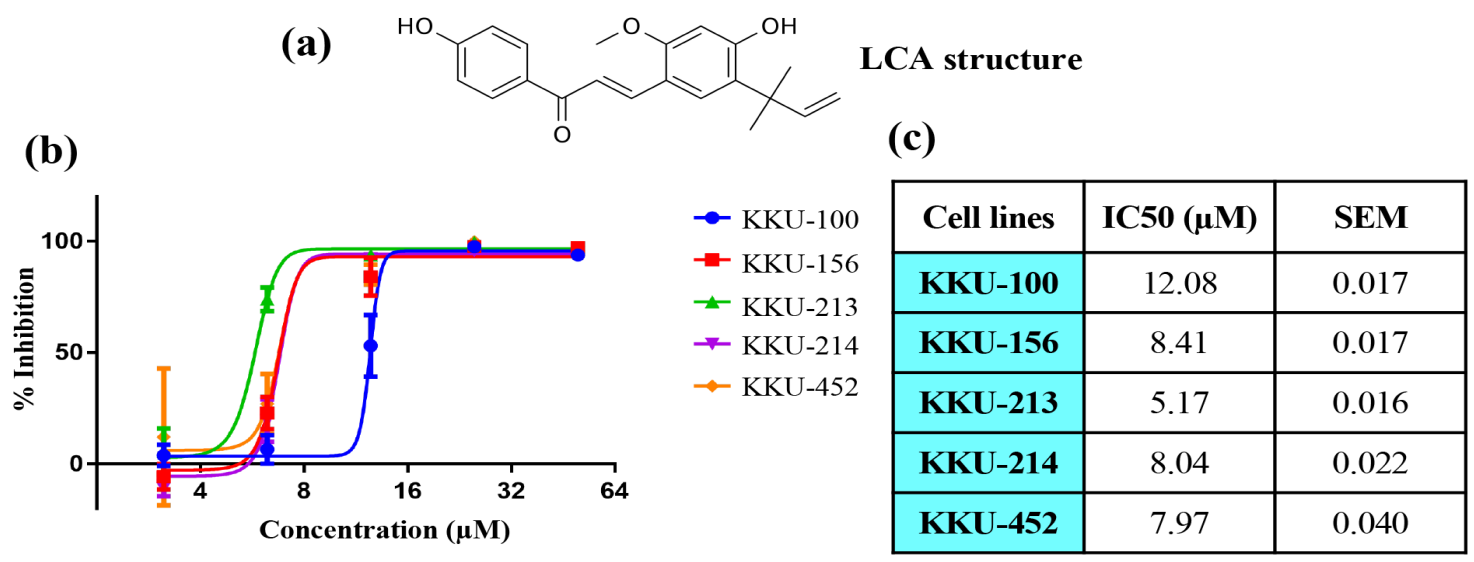

Figure 1. Inhibitory Effect of Licochalcone A on Cholangiocarcinoma Cells. (a), Chemical structure of licochalcone A (LCA); (b), Cholangiocarcinoma (CCA) cell lines were exposed to various concentrations of LCA for 72 hours. Cells were fixed and stained for cytotoxicity. Graphs indicate percentage inhibition curve versus logarithmic values of LCA concentrations; (c), Table summarized half-maximal inhibitory concentration $\left(\mathrm{IC}_{50}\right)$ of LCA on CCAs and standard errors of each cell. 

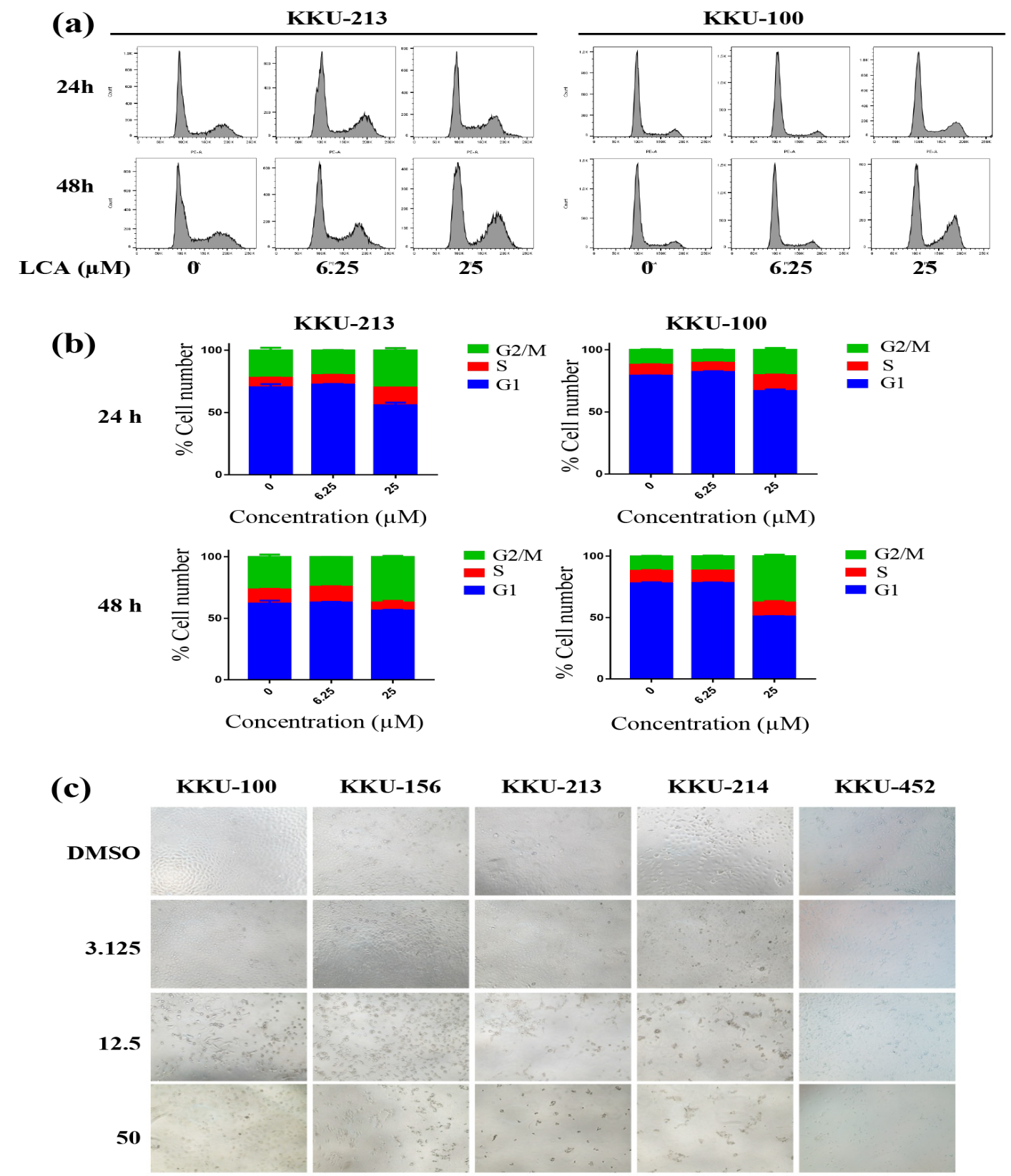

Figure 2. Licochalcone A induces G2/M Arrest and Apoptotic Cell Death. (a), Histogram plots indicate cell cycle analysis of CCAs after exposure to LCA $(0,6.25$, and $25 \mu \mathrm{M})$ for 24 and 48 hour; (b) Stacked bars show proportion of cells \pm SEM in each cell cycle phase after treatment of LCA at indicated concentrations; (c), Bright field images at 4x magnification show cellular morphology of CCAs after exposure to various concentrations of LCA for 72 hours.

of the mitochondrial-mediated cell death pathway (Figure $4 \mathrm{a}, \mathrm{b}$ ).

\section{Licochalcone $A$ induces reactive oxygen species accumulation}

To study the mechanism of cytotoxicity of LCA, the reactive oxygen species (ROS) were first focused upon. The redox state of the cells plays a critical role in cellular processes which include cell proliferation, migration, differentiation and death (Tochhawng et al., 2012; Panieri and Santoro, 2016). To detect the ROS level, after exposure with LCA for $24 \mathrm{~h}$, CCA cells were stained with an ROS-sensitive fluorescent probe, dihydroethidium (DHE). Figure 5a illustrates the ROS buildup intracellularly in CCA cells as shows by bright red fluorescence color upon treatment of LCA in a dosedependent manner (Figure $5 \mathrm{~b}$ ). These results indicates that LCA could trigger ROS accumulation and was associated with cell death.

\section{LCA suppresses Nrf2 and antioxidant enzymes}

Accumulation of ROS is developed from various conditions. Overproduction of ROS or ablation of the ROS scavenging system causes a redox imbalance that leads to oxidative stress and cell death. The study then explored whether LCA-induced ROS and cell death resulted from a defect in the antioxidant and cytoprotective system. The experiment was carried out to determine the expression of nuclear factor-erythroid factor 2-related factor 2 (Nrf2), a critical transcription factor that is responsible 


\section{(a)}
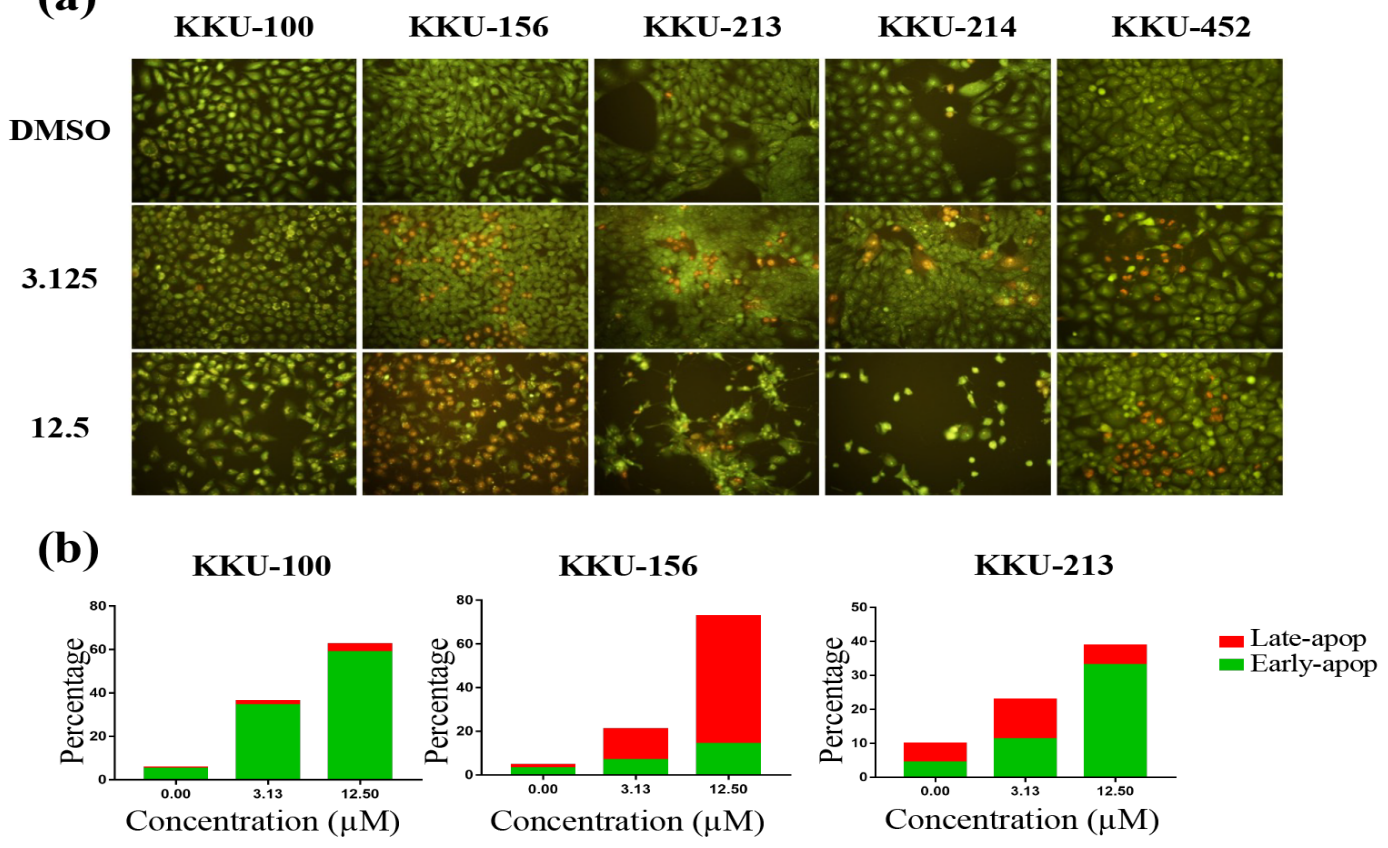

KKU-214
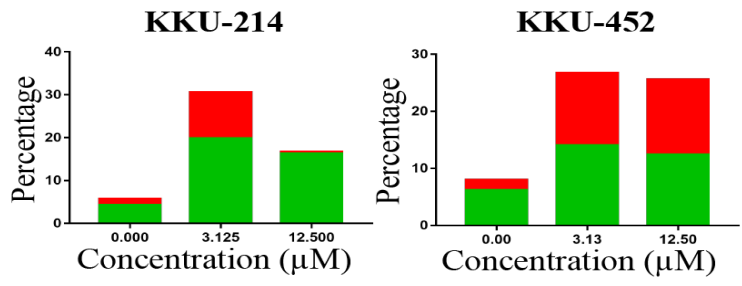

Late-apop
Early-apop

Figure 3. LCA Induced Apoptosis Cell Death. (a), Acridine orange/ethidium bromide staining indicating cell death. Early apoptotic cells appear in bright green fluorescence while late apoptotic cells appear in orange fluorescence after LCA treatment 72 hours at 10x magnification; (b), Bars indicate percentage from total cells of early apoptotic and late apoptotic cells.

(a)

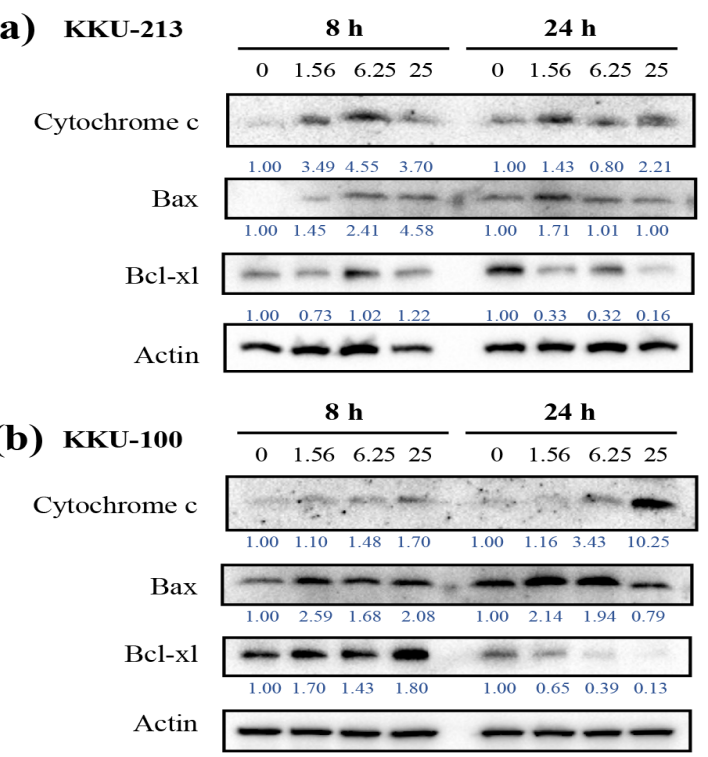

Figure 4. LCA Induced Mitochondrial-Mediated Cell Death. (a), Western blot (WB) analysis of apoptotic proteins (Cytochrome c, Bax, and Bcl-xL) expression in KKU-213 after treatment with LCA for ( 8 and 24 hour). Actin was used as a loading control. The number under each blot indicates normalized signals to loading control; (b), Western blot (WB) analysis of same condition in Figure $3 \mathrm{~A}$ in KKU-100. for redox regulation. It was found that the expression of Nrf2 was decreased in KKU-100 cells after 8-24 h of incubation that coincided with the ROS level that was upregulated. However, the level of Nrf2 was unchanged in KKU-213 cells. Beside the Nrf2, heme oxygenease-1 (HO-1), and Glutamate-Cysteine Ligase Catalytic Subunit (GCLC) expression were also suppressed, particularly in KKU-100 cells indicating a decline in antioxidant defense and cytoprotective activities in CCA cells (Figure 6a).

\section{Migratory inhibition effect of licochalcone A}

To investigate if LCA could inhibit migration of cancer cells, the wound healing assay was used to determine the anti-migration effect of LCA. Cell migration kinetics were examined under microscopy after being treated with LCA at $0,24,48$ and $72 \mathrm{~h}$. The results showed that control KKU-100 cells migrated across the wound and closed the wound gap by $90 \%$ at $72 \mathrm{~h}$. LCA showed a strong anti-migration activity; even at the lowest concentration, LCA could suppress CCA cells migration significantly (Figure 7a, b).

Since anti-migration by LCA was apparent at low cytotoxic concentration, a further experiment was to examine the effects of LCA on NF- $\mathrm{KB}$-mediated cell migration. $\mathrm{NF}-\kappa \mathrm{B}$ protein expression was determined 


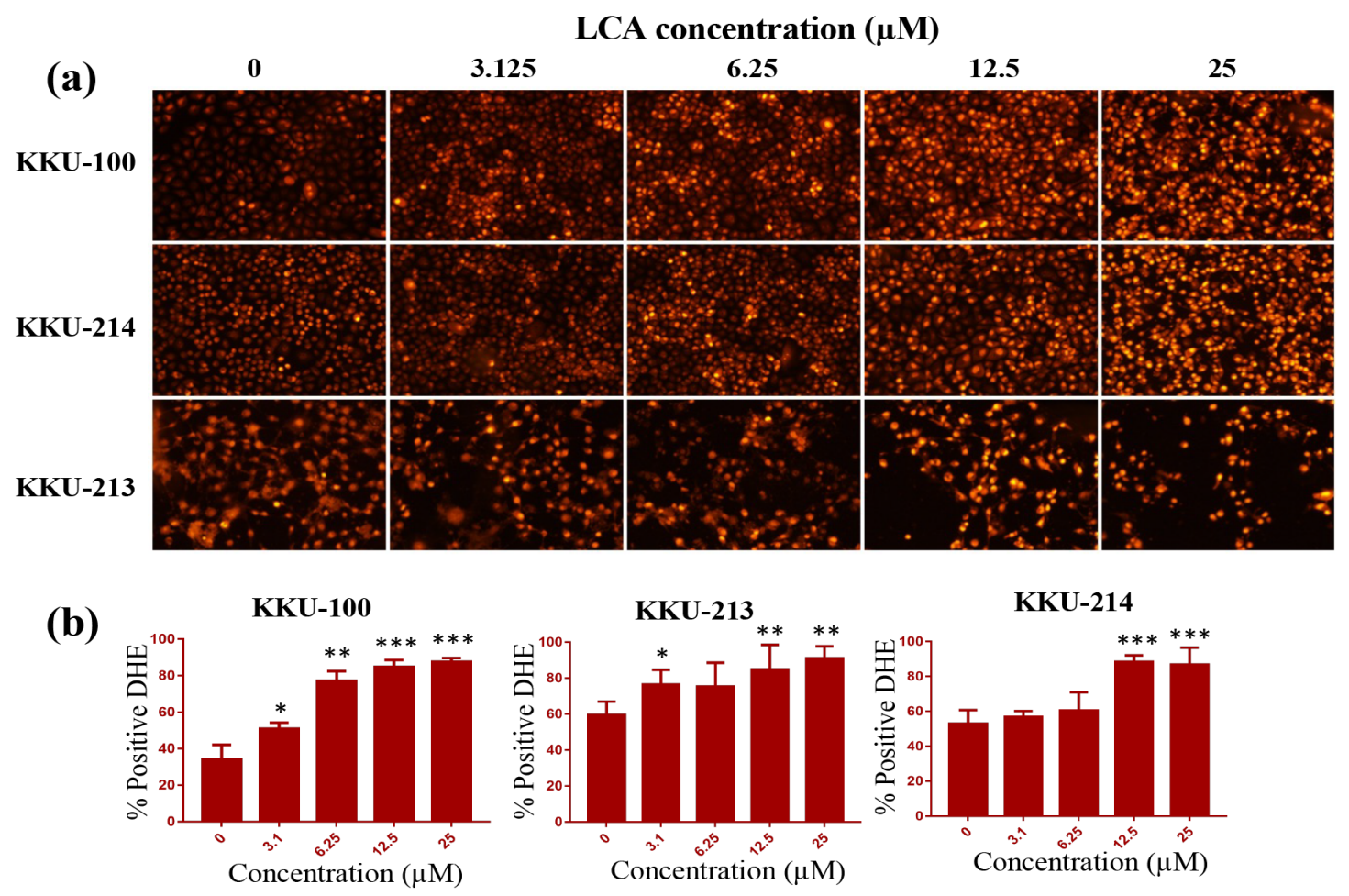

Figure 5. CCA Cells Accumulate Higher Levels of ROS when Exposed to LCA. (a), Reactive oxygen species accumulation in CCAs (KKU-100, KKU-213, KKU-214) after 24-hour LCA treatment designated by DHE fluorescence dye staining. Positive cells are shown in bright red fluorescence; (b), Bars represent the average percentages of positive DHE cells compared to total cell numbers + s.d. from triplicated experiments. Statistical significance was determined by ANOVA with multiple comparisons (Dunnett's test) between vehicle control $(0 \mu \mathrm{M})$ and indicated concentrations of LCA. $\left({ }^{*} \mathrm{p} \leq 0.05, * * \mathrm{p} \leq 0.01\right.$ and $\left.* * * \mathrm{p} \leq 0.001\right)$.
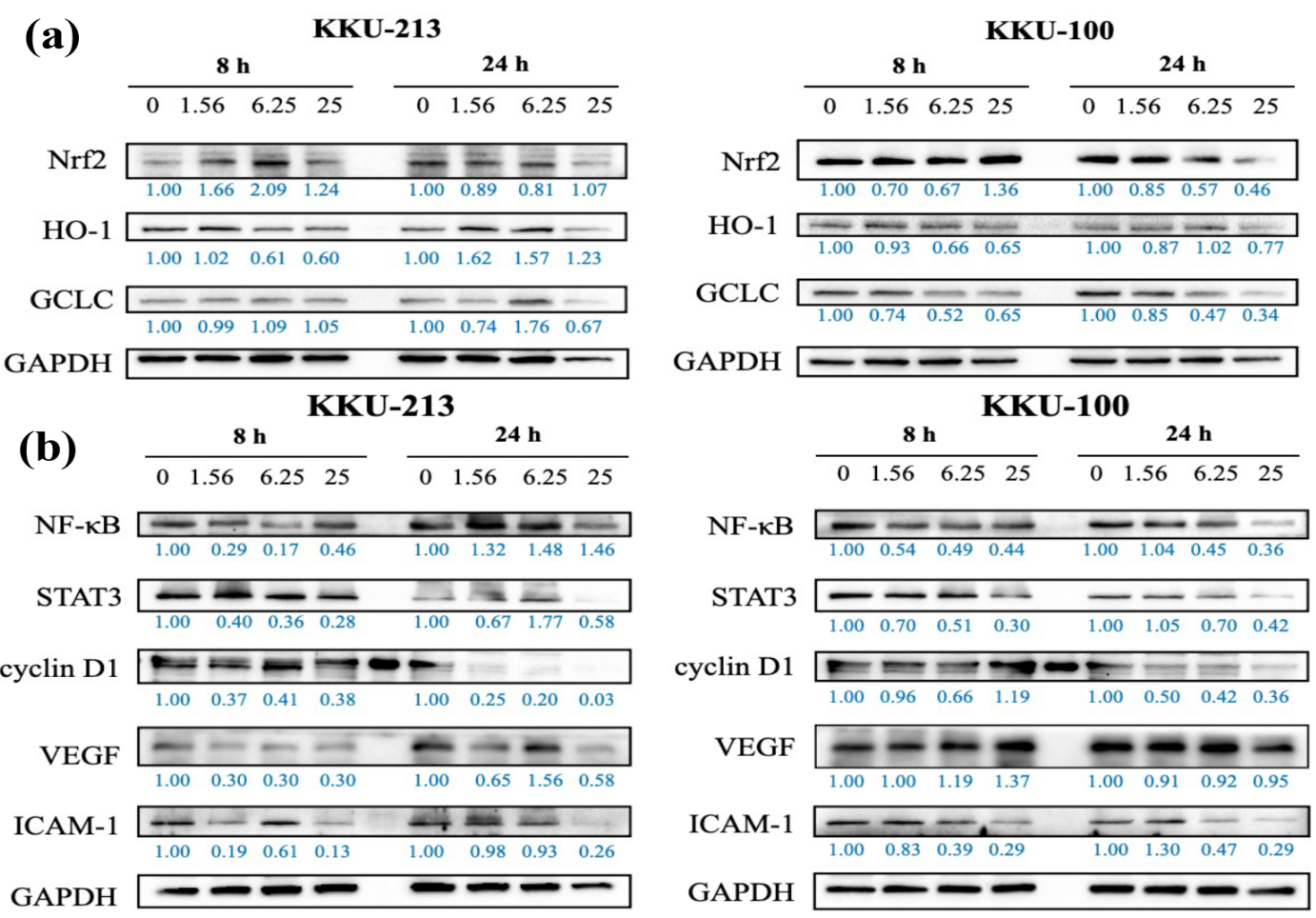

Figure 6. LCA Suppresses Antioxidant Nrf2, NF-кB, and STAT3 Pathway. (a), Western blot (WB) analysis of Nrf2 pathway proteins (Nrf2, HO-1, and GCLC) expression of KKU-213 and KKU-100 after treatment by LCA for 8 and 24 hours. GAPDH was used as a loading control. The number under each blot indicates normalized signals to the loading control; (b), Western blot (WB) analysis of NF- $\kappa$ B pathway proteins (NF- $\kappa$ B, STAT3, cyclin D1, VEGF, and ICAM-1) expression in KKU-213 and KKU-100 after treatment of LCA for 8 and 24 hours. GAPDH was used as a loading control. The number under each blot indicates normalized signals to loading control. 
(a)

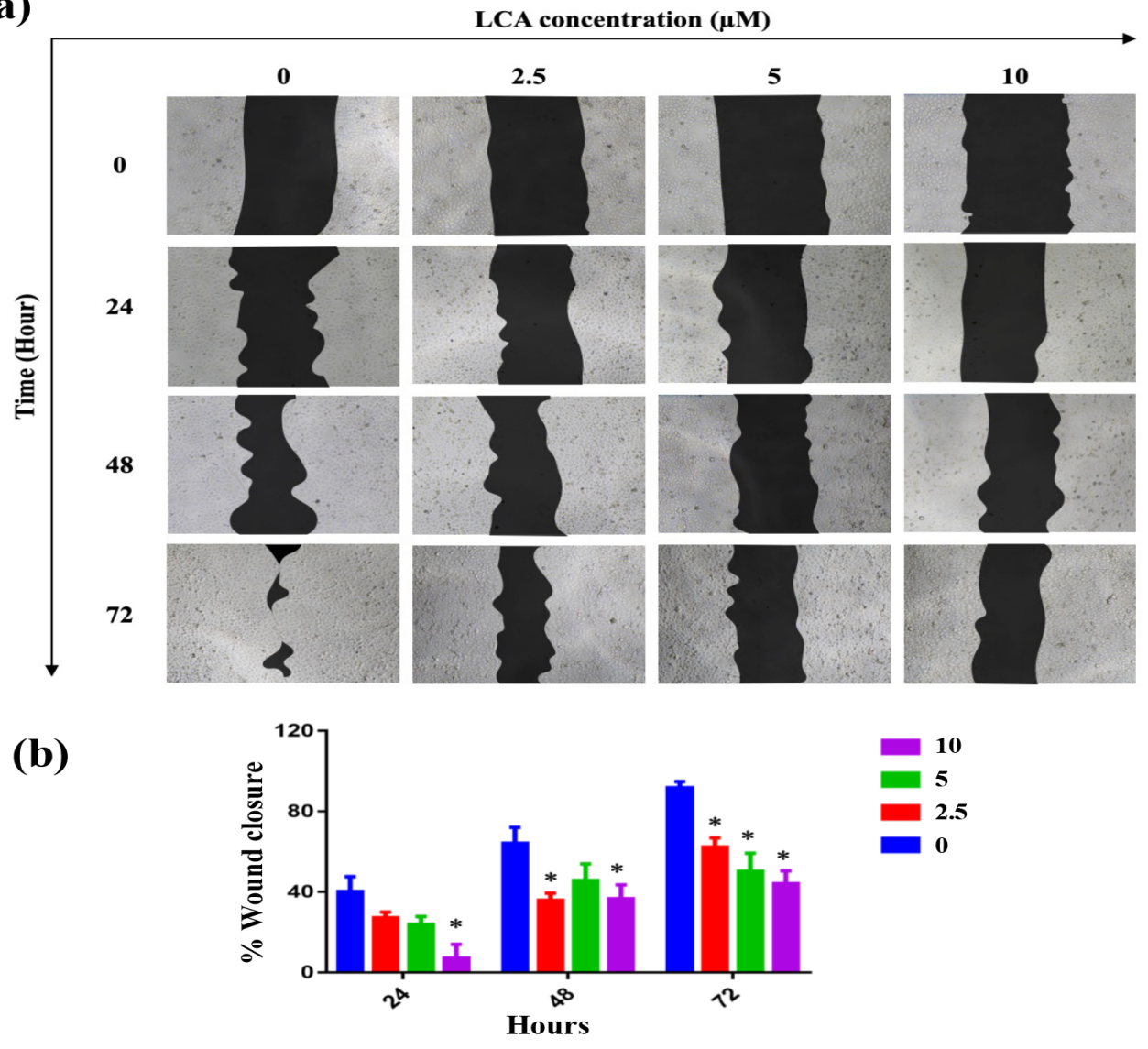

Figure 7. LCA Inhibits Cholangiocarcinoma Migration. (a), Wound healing assay at 0, 24, 48, and 72 hours with LCA treatment at 2.5, 5, and $10 \mu \mathrm{M}$; (b), Graphs show percent wound closure + SEM. Statistical significance was determined by two-way ANOVA $\left({ }^{*} \mathrm{P} \leq 0.05\right.$, at 24 hour of 0 vs $10 \mu \mathrm{M}$, at 48 hour of 0 vs $2.5,10 \mu \mathrm{M}$, at 72 hour of 0 vs $2.5,5,10 \mu \mathrm{M})$.

and it was found that the protein was rapidly decreased upon treatment with LCA. Moreover, its cooperative partner in inflammation and cancer progression, the signal transducer and activator of transcription 3 (STAT3) was also depleted. Transactivation of NF- $\mathrm{KB}$ and STAT3 was down-regulated and evaluation of their downstream target proteins including cyclin D1, VEGF, Bcl-xL and the intercellular adhesion molecule-1 (ICAM-1) protein especially at $24 \mathrm{~h}$ were decreased in both cells (Figure $6 \mathrm{~b}$ ). The result suggested that LCA suppressed NF-кB-STAT3mediated CCA cell migration through inhibition of its downstream expression.

\section{Discussion}

A flavonoid-based chalcone compound, licochalcone A (LCA), has been reported to have a wide range of pharmacological effects against various human diseases. The well-established examples for the effects of LCA are anti-inflammation, anti-oxidative stress, anti-microorganisms, anti-aging and anti-cancer activities (Fu et al., 2013; Gatta Daniela Maria et al., 2019; Ze-Feng et al., 2020). The present study showed that LCA potently inhibited CCA cell proliferation and cell migration. The effects were associated with ROS formation, suppression of Nrf2, NF- $\mathrm{B}, \mathrm{STAT} 3$ and antioxidant enzymes and pro-metastatic proteins.

The results herein showed that LCA suppressed expression of Nrf2 and its downstream enzymes including HO-1, a powerful cytoprotective enzyme (Prawan et al. 2005) and GCLC, the rate limiting enzyme in the synthesis of GSH (Krzywanski et al., 2004). The Nrf2 suppression was found obviously in KKU-100. Nrf2 plays critical roles in regulation of cellular redox, antioxidant defense and intermediary metabolism (Hayes and Dinkova-Kostova et al., 2014). Cancer cells also exploit Nrf2 for cell survival and confer resistance to the harsh tumor microenvironment and chemotherapeutic agents (Hayashi et al., 2020). Therefore, suppression of Nrf2 may diminish the cellular protective mechanisms and render cancer more vulnerable to therapeutic agents. For KKU-213 cells, Nrf2 expression was unchanged, it was probably that LCA may induce cell death via other mechanisms including NF- $\kappa B$. This may correspond to a low induction of ROS in KKU-213 cells compared to KKU-100 (Figure 5a, b).

An increase in ROS formation may be resulted from the inhibition of Nrf2- downstream antioxidant and cytoprotective enzymes such as NQO1, HO-1, GCLC, GCLM, GPX, GST, Trx etc (Hayes and Dinkova-Kostova et al., 2014). Excessive ROS accumulation may cause an imbalance of the redox state with a consequence of interfering with cellular functions and if it exceeds the 
cellular protection threshold it can lead to a trigger of cell death. In KKU-100 cells, ROS formation was associated with decreased Bcl-xL, increased Bax and cytochrome $\mathrm{c}$ expression. Moreover, the increased cytochrome $\mathrm{c}$ and Bax were also observed in KKU-213 cells. This implies that LCA-induced cell death is mediated via the mitochondrial cell death pathway in CCA cells through Nrf2 and nonNrf2 pathways.

Antitumor activities of LCA have been demonstrated in various cancer types both in vitro and in vivo. LCA has reportedly suppressed cancer cell proliferation through inhibition of cell cycle progression at different phases of the cell cycle suggesting that LCA may have a distinct mechanism in each type of cell (Lu et al., 2018; Wang et al., 2018; Hong et al., 2019). Most of the studies showed that LCA arrested cancer cells in G2/M phase as a consequence of ROS induction (Wang et al., 2018; Hong et al., 2019). This study was also consistent with the effect that cell arrest at $\mathrm{G} 2 / \mathrm{M}$ phase.

Here it was found that LCA suppressed both $\mathrm{NF}-\kappa \mathrm{B}$ protein expression and activities as judged by the decreased expression of its downstream regulated genes including various survival-regulator proteins; Bcl-xL, cyclin D1, VEGF and ICAM-1. This NF- $\mathrm{B}$ suppression was found in both KKU-213 and KKU-100. This is consistent with reports that LCA inhibited colon cancer cell proliferation and promoted apoptosis by the NF- $\kappa \mathrm{B}$ and Ras-MAPK pathways (Liu et al., 2021). NF- $\kappa$ B plays important role in natural and adaptive immunity, and cancer development in which NF- $\mathrm{BB}$ is activated by inflammatory cytokines (DiDonato et al., 2012). Another key regulator that is working for the inflammation cascade and stress response pathway that intertwines with NF- $\kappa$ B is STAT3. In addition, STAT3 was also down regulated concurrently with NF- $\mathrm{KB}$ pathway inhibition upon LCA treatment. JAK/STAT3 signaling is involved in the development of several cancers including CCA (Simile et al., 2019). NF-кB and STAT3 play an important role in metastasis of cancer by regulating metastatic proteins including ICAM-1 and VEGF (Bollrath and Greten, 2009). ICAM-1 is essential for cancer cell invasion and metastasis. This explains the effects of LCA not only induces CCA cell death, but also inhibits cell migration and probably metastasis.

The ability of cancer to migrate from the primary tumor site to the surrounding tissue is a major characteristic of aggressive and metastatic cancer. Previous studies reported that each cholangiocarcinoma cell has different basal redox status (Laphanuwat et al., 2018). The complexities behind individual pathohistological type of CCA might give rise to a distinct stress responsive pathway. Here, it was found that KKU-213 has a higher basal ROS compared to KKU-100 (Figure 5b, control groups) that coincided with the higher cell death response following exposure to LCA. The response to the Nrf2 pathway suppression predominantly occurred in KKU-100, while $\mathrm{NF}-\kappa \mathrm{B}$ suppression was found in both cells. The responses of these two CCA cell lines toward stresses indicated that each cancer cell line has a different way to adapt itself to the overwhelming oxidative stage, as a result, rewiring their stress response pathway (LeBoeuf et al., 2020). Taken together, the dual suppression of both Nrf2 and NF- $\kappa$ B by LCA is likely to be a valuable mechanism in inhibition of the resistant cancer cells like a one-two punch strategy.

Cholangiocarcinomas is a lethal cancer that currently lacks effective therapy. LCA-induced CCA cells death demonstrated the promising possible outcome for using this compound for CCA treatment. Further investigation in vivo of how CCA tumors response to the LCA treatment and what could be the most suitable biomarker for the prediction of CCA responses is required to confirm the efficiency of this compound.

\section{Author Contribution Statement}

PL conceptualized this project. PL designed and performed the experiments. PL and VK analyzed the data. PL, SK, AP, LS and VK for critical comments. PL and VK wrote manuscript. All authors approved the final manuscript.

\section{Acknowledgements}

We would like to acknowledge Prof. James Arthur Will, for editing the MS via Publication Clinic KKU, Thailand.

\section{Funding}

This research was supported by the Basic Research Fund of Khon Kaen University under Cholangiocarcinoma Research Institute

\section{Statement conflict of Interest}

The authors declare no competing or financial interests

\section{References}

Al-Rajabi R, Sun W (2021). Immunotherapy in cholangiocarcinoma. Curr Opin Gastroenterol, 37, 105-111.

Banales JM, Marin JJG, Lamarca A, et al (2020). Cholangiocarcinoma 2020: the next horizon in mechanisms and management. Nat Rev Gastroenterol Hepatol, 17, 557-88.

Bollrath J, Greten FR (2009). IKK/NF-kappaB and STAT3 pathways: central signalling hubs in inflammation-mediated tumour promotion and metastasis. EMBO Rep, 10, 1314-9.

Chen G, Ma Y, Jiang Z, et al (2018). Lico A causes ER stress and apoptosis via up-regulating miR-144-3p in human lung cancer cell line H292. Front Pharmacol, 9, 837.

DeNicola GM, Karreth Fa Fau - Humpton TJ, Humpton Tj Fau - Gopinathan A, et al (2011). Oncogene-induced Nrf2 transcription promotes ROS detoxification and tumorigenesis. Nature, 475, 106-9.

DiDonato JA, Mercurio F Fau, Karin M (2012). NF-kB and the link between inflammation and cancer. Immunol Rev, 246, 379-400.

Doherty B, Nambudiri VE, Palmer WC (2017). Update on the Diagnosis and Treatment of Cholangiocarcinoma. Curr Gastroenterol Rep, 19, 2.

Fu Y, Chen J, Li Y-J, et al(2013). Antioxidant and anti-inflammatory activities of six flavonoids separated from licorice. Food Chem, 141, 1063-71.

Gatta Daniela Maria P, Franceschelli S, Felaco M, et al (2019). Biological Effects of Licochalcones. Mini Rev Med Chem, 
19, 647-56.

Hayashi M, Kuga A, Suzuki M, et al (2020). Microenvironmental activation of $\mathrm{Nrf2}$ restricts the progression of Nrf2-activated malignant tumors. Cancer Res, 80, 3331-44.

Hayes JD, Dinkova-Kostova AT (2014). The Nrf2 regulatory network provides an interface between redox and intermediary metabolism. Trends Biochem Sci, 39, 199-218.

Hong SH, Cha H-J, Hwang-Bo H, et al (2019). Anti-proliferative and pro-apoptotic effects of licochalcone a through ROSmediated cell cycle arrest and apoptosis in human bladder cancer cells. Int J Mol Sci, 20.

Khan SA, Tavolari S, Brandi G (2019). Cholangiocarcinoma: Epidemiology and risk factors. Liver Int, 39, 19-31.

Krzywanski DM, Dickinson DA, Iles KE, et al (2004). Variable regulation of glutamate cysteine ligase subunit proteins affects glutathione biosynthesis in response to oxidative stress. Arch Biochem Biophys, 423, 116-25.

Kubo S, Takemura S, Tanaka S, et al (2017). Occupational cholangiocarcinoma caused by exposure to 1,2-dichloropropane and/or dichloromethane. Ann Gastroenterol Surg, 2, 99-105.

Laphanuwat P, Likasitwatanakul P, Sittithumcharee G, et al (2018). Cyclin D1 depletion interferes with oxidative balance and promotes cancer cell senescence. J Cell Sci, 131.

LeBoeuf SE, Wu WL, Karakousi TR, et al (2020). Activation of oxidative stress response in cancer generates a druggable dependency on exogenous non-essential amino acids. Cell Metab, 31, 339-50.e4.

Lee CH, Chang CJ, Lin YJ, et al (2009). Viral hepatitisassociated intrahepatic cholangiocarcinoma shares common disease processes with hepatocellular carcinoma. $\mathrm{Br} J$ Cancer, 100, 1765-70.

Liu D, Zhang L, Duan L, et al (2019). Potential of herb-drug / herb interactions between substrates and inhibitors of UGTs derived from herbal medicines. Pharmacol Res, 150, 104510

Liu X, Xing Y, Li M, et al (2021). Licochalcone A inhibits proliferation and promotes apoptosis of colon cancer cell by targeting programmed cell death-ligand 1 via the NF- $\mathrm{\kappa B}$ and Ras/Raf/MEK pathways. J Ethnopharmacol, 273.

Lu WJ, Wu GJ, Chen RJ, et al (2018). Licochalcone A attenuates glioma cell growth in vitro and in vivo through cell cycle arrest. Food Funct, 9, 4500-7.

Lv H, Yang H, Wang Z, et al (2019). Nrf2 signaling and autophagy are complementary in protecting lipopolysaccharide/dgalactosamine-induced acute liver injury by licochalcone A. Cell Death Dis, 10, 313.

Panieri E, Santoro MM (2016). ROS homeostasis and metabolism: a dangerous liason in cancer cells. Cell Death Dis, 7, e2253-e.

Prawan A, Kundu Jk Fau - Surh Y-J, Surh YJ (2005). Molecular basis of heme oxygenase-1 induction: implications for chemoprevention and chemoprotection. Antioxid Redox Signal, 7, 1688-703.

Raj L, Ide T, Gurkar AU, et al (2011). Selective killing of cancer cells by a small molecule targeting the stress response to ROS. Nature, 475, 231-4.

Shen H, Zeng G Fau - Tang G, Tang G Fau - Cai X, et al (2014). Antimetastatic effects of licochalcone A on oral cancer via regulating metastasis-associated proteases. Tumour Biol, 35, 7467-74

Simile MM, Bagella P, Vidili G, et al (2019). Targeted therapies in cholangiocarcinoma: Emerging Evidence from Clinical Trials. Medicina, 55.

Tochhawng L, Deng S, Pervaiz S, et al (2012). Redox regulation of cancer cell migration and invasion. Mitochondrion, 13.

Tyson GL, El-Serag HB (2011). Risk factors for cholangiocarcinoma. Hepatology, 54, 173-84.

Wang J, Zhang Y-S, Thakur K, et al (2018). Licochalcone A from licorice root, an inhibitor of human hepatoma cell growth via induction of cell apoptosis and cell cycle arrest. Food Chem Toxicol, 120, 407-17.

Yue PYK, Leung EPY, Mak NK, et al (2010). A simplified method for quantifying cell migration/wound healing in 96-well plates. J Biomol Screen, 15, 427-33.

Zabron A, Edwards RJ, Khan SA (2013). The challenge of cholangiocarcinoma: dissecting the molecular mechanisms of an insidious cancer. Dis Model Mech, 6, 281-92.

Ze-Feng W, Jia L, Yong-An Y, et al (2020). A Review: The anti-inflammatory, anticancer and antibacterial properties of four kinds of licorice flavonoids isolated from licorice. Curr Med Chem, 27, 1997-2011.

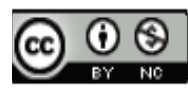

This work is licensed under a Creative Commons AttributionNon Commercial 4.0 International License. 